$1-1-2012$

\title{
Larynx-sparing techniques using intensity-modulated radiation therapy for oropharyngeal cancer.
}

\author{
Voichita Bar-Ad \\ Thomas Jefferson University \\ Haibo Lin \\ University of Pennsylvania \\ Wei-Ting Hwang \\ University of Pennsylvania \\ Curtiland Deville \\ University of Pennsylvania \\ Pinaki R Dutta \\ University of Pennsylvania \\ Follow this and additional works at: https://jdc.jefferson.edu/radoncfp \\ Part of the Oncology Commons

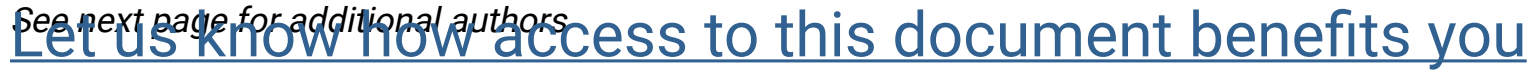

\section{Recommended Citation}

Bar-Ad, Voichita; Lin, Haibo; Hwang, Wei-Ting; Deville, Curtiland; Dutta, Pinaki R; Tochner, Zelig; and Both, Stefan, "Larynx-sparing techniques using intensity-modulated radiation therapy for oropharyngeal cancer." (2012). Department of Radiation Oncology Faculty Papers. Paper 25. https://jdc.jefferson.edu/radoncfp/25

This Article is brought to you for free and open access by the Jefferson Digital Commons. The Jefferson Digital Commons is a service of Thomas Jefferson University's Center for Teaching and Learning (CTL). The Commons is a showcase for Jefferson books and journals, peer-reviewed scholarly publications, unique historical collections from the University archives, and teaching tools. The Jefferson Digital Commons allows researchers and interested readers anywhere in the world to learn about and keep up to date with Jefferson scholarship. This article has been accepted for inclusion in Department of Radiation Oncology Faculty Papers by an authorized administrator of the Jefferson Digital Commons. For more information, please contact: JeffersonDigitalCommons@jefferson.edu. 


\section{Authors}

Voichita Bar-Ad, Haibo Lin, Wei-Ting Hwang, Curtiland Deville, Pinaki R Dutta, Zelig Tochner, and Stefan Both 


\section{As submitted to:}

Medical Dosimetry

And later published as:

Larynx-Sparing Techniques using Intensity-Modulated

Radiation Therapy for Oropharyngeal Cancer

Volume 37, Issue 4, 2012, Pages 383-386

DOI: 10.1016/j.meddos.2012.02.004

Voichita Bar Ad, M.D.,

Haibo Lin, Ph D. ${ }^{1}$

Wei-Ting Hwang, Ph D. ${ }^{2}$

Curtiland Deville, M.D. ${ }^{1}$

Pinaki R. Dutta, M.D., Ph D.,"\#

Zelig Tochner, M.D. ${ }^{1}$

Stefan Both, Ph D. ${ }^{1}$

${ }^{1}$ Department of Radiation Oncology, University of Pennsylvania, Philadelphia, PA

${ }^{2}$ Department of Biostatistics and Epidemiology, University of Pennsylvania, Philadelphia,

$\mathrm{PA}$

${ }^{*}$ Current address: Department of Radiation Oncology, Thomas Jefferson University,

Philadelphia, PA

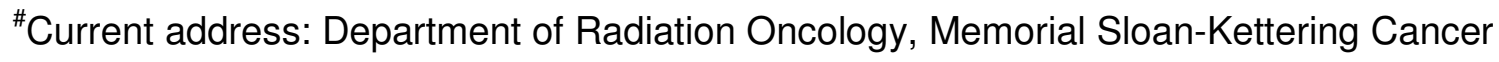


Center, Rockville Centre, NY

Correspondence and reprint requests to:

Voichita Bar Ad, M.D.

Department of Radiation Oncology

Thomas Jefferson University

Philadelphia, PA 19107

Phone: 215-955-5936

Fax: 215-503-0013

E-mail: Voichita.Ba-Ad@Jeffersonhospital.org

Presented in part at American Society of Therapeutic Radiology and Oncology annual meeting, SanDiego, October 31st -November 5th, 2010.

Running title: larynx-sparing technique using IMRT

Disclosure: No conflict of interest. No funds to declare.

Keywords: laryngeal dose, IMRT, non-laryngeal head and neck cancer

\section{ABSTRACT}

Purpose: The purpose of the current study was to explore if the laryngeal dose can be reduced by using two Intensity-Modulated Radiation Therapy (IMRT) techniques: whole neck field IMRT technique (WF-IMRT) vs. junctioned IMRT (J-IMRT). The effect on planning target volumes (PTVs) coverage and laryngeal sparing was evaluated. 
Method: WF-IMRT technique consisted of a single IMRT plan including the primary tumor, the superior and inferior neck to the level of the clavicular heads. The larynx was defined as an organ at risk extending superiorly to cover the arythenoid cartilages and inferiorly to include the crycoid cartilage. The J-IMRT technique consisted of an IMRT plan for the primary tumor and the superior neck, matched to a conventional antero-posterior opposing lower neck fields at the level of the thyroid notch. A central block was used for the anterior lower neck field at the level of the larynx in order to restrict the dose to the larynx. Ten oropharyngeal cancer cases were analyzed. Both the primary site and bilateral regional lymphatics were included in the radiotherapy targets.

Results: The averaged V95 for the PTV57.6 was $99.2 \%$ for WF-IMRT technique when compared with $97.4 \%(p=0.02)$ for J-IMRT. The averaged V95 for the PTV64 was $99.9 \%$ for WF-IMRT technique when compared with 98.9\% $(\mathrm{p}=0.02)$ for J-IMRT and the averaged V95 for the PT70 was $100.0 \%$ for WF-IMRT technique when compared with $99.5 \%(p=0.04)$ for J-IMRT. The averaged mean laryngeal dose was 18 Gy with both techniques. The averaged mean dose within the matchline volumes were 69.3Gy for WF-MRT vs 66.2 Gy for J-IMRT $(p=0.03)$. Conclusion: WF-IMRT technique appears to offer an optimal coverage of the target volumes and a mean dose to the larynx similar with J-IMRT and should be further evaluated in clinical trials.

\section{INTRODUCTION}

The optimal management of oropharyngeal cancer is multidisciplinary and radiotherapy is a part of treatment approach for most of these patients. The greatest challenge with radiotherapy for oropharyngeal cancer is to maximize loco-regional disease control, while minimizing the morbidity and treatment-related toxicity to surrounding normal tissues, including the larynx [1]. With the development of the Intensity-Modulated Radiation Therapy (IMRT) technique, head and neck cancers have been treated with good results as reported by various investigators $[2,3]$. 
IMRT has been shown to offer better target homogeneity and tumor coverage and normal organ sparing [3,4]. However, the use of IMRT tends to unnecessarily deliver higher dose to the larynx [5]. More than one decade ago, Mendenhall et al. discouraged the practice of including the uninvolved larynx in the radiation fields when the non-laryngeal primary tumor site could be adequately covered by placing the inferior border of the opposed lateral fields at the level of the thyroid notch. The superior section of the plan was matched to a conventional anterior neck field resulting in very low doses to the larynx, which was typically blocked on the anterior field [1]. Emerging data from patients with long term follow up have showed that irradiation of the normal larynx is associated with laryngeal dysfunction affecting patients' quality of life in head and neck cancer survivors, treated with radiation therapy [6,7]. Furthermore, recent studies by Eisburch et al. have shown that sparing larynx improves dysphagia and aspiration [8].

The current study was undertaken to determine if the laryngeal dose can be reduced when using IMRT techniques including the attempt to spare the larynx based on given dose constraints for this organ at risk when a whole-neck field IMRT (WF-IMRT) is chosen or the use of a junctioned IMRT (J-IMRT) consisting of an IMRT plan for the primary non-laryngeal tumor and the superior neck, matched with a conventional opposed antero-posterior lower neck fields at the level of the thyroid notch.

\section{MATERIALS \& METHODS}

\section{Patient Selection}

Ten consecutive patients diagnosed with stage IV oropharyngeal squamous cell carcinoma and treated between September 2006 and January 2007, at the University of Pennsylvania, Department of Radiation Oncology were included in the current study. Approval was granted by the Institutional Review Board for conducting this study. Nine patients were diagnosed with stage IV A disease (T1-3N2bM0) and one patient with stage IV B disease (T2N3M0). The nodal 
disease at the neck in four cases extended inferiorly to the level of the glottic larynx. All patients were treated with IMRT to the primary site and the bilateral cervical lymph nodes. Two treatment plans were generated for each patient using WF-IMRT and J-IMRT techniques and the dosimetric data was compared between the two treatment plans. The coverage of the target volumes and the dose to the larynx were compared using the resulting dose volume histograms and the following dose parameters: V100 and V95 for the targets, and mean laryngeal dose. V100 was defined as the percent of the target volume covered by $100 \%$ of the prescribed dose and V95 was defined as the percent of the target volume covered by $95 \%$ of the prescribed dose.

Radiotherapy treatment planning

All patients underwent a CT-based planning for IMRT using a $3 \mathrm{~mm}$ CT slice thickness to cover the oropharyngeal tumors and bilateral cervical lymph nodes. Gross tumor volume (GTV) was defined as the grossly visible tumor and metastatic lymphadenopathy on physical examination and imaging studies (contrast CT and/or MRI). Clinical target volume (CTV1) encompassed the GTV and the adjacent tissue supposed to contain microscopic, subclinical tumor extension and first echelon nodes. The uninvolved nodal areas that were at lower risk of subclinical disease, treated prophylactically were included in the CTV2 volume. Planning target volume (PTV) was obtained by expansion of the CTVs to compensate for setup uncertainty, typically by 3-5 mm. The PTV was adjusted appropriately, to respect the anatomical bounders located in close proximity to the target volumes. Briefly, 70.4 Gy in 32 fractions were prescribed to the PTVs of the gross disease (PTV70.4), 64 Gy in 32 fractions to the PTVs of high risk subclinical disease (subclinical disease at the surrounding area of the primary tumor and first echelon lymph nodes) (PTV64), and 57.6 in 32 fractions to the PTVs of the lesser risk sublicnical disease (the rest of the neck nodal targets) (PTV57.6), respectively. All patients in this group were treated with a simultaneous in-field boost IMRT 
technique. Inverse treatment planning was performed using the treatment planning system Eclipse v.8.10, anisotropic analytical algorithm (AAA). For each patient, a plan with 7-9 coplanar sliding-window IMRT beams of 6MV was generated. The J-IMRT technique consisted of an IMRT plan for the primary tumor and the superior neck above the level of the arythenoids. The lower neck and the supraclavicular fossae were treated using a conventional half-beam blocked antero-posterior opposing neck fields matched with the upper neck IMRT plan at the level of the thyroid notch. A central block was used for the anterior lower neck field at the level of the larynx in order to restrict the dose to this organ at risk, and a full central cord block was used for the posterior field to protect the spinal cord. The upper neck IMRT was matched with the lower neck field using a split-beam technique and there was no gap between the superior IMRT fields and the inferior neck fields. Matchline volumes have been defined to include $3 \mathrm{~mm}$ bellow and above the actual matchline for the PTVs and dose distribution has been evaluated in this region. WF-IMRT technique consisted of a single IMRT plan including the primary tumor, the superior and inferior neck to the level of the clavicular heads, avoiding the matchline level present with J-IMRT technique. The larynx was considered an organ of risk and was defined as superiorly covering the arythenoid cartilages and inferiorly including the cricoid cartilage. The lateral borders of the planning organ at risk volume (PRV) of the larynx were contoured 3-5 mm away from the medial borders of the neck PTVs in order to avoid underdosage in these areas of the target volumes. Other organs at risk included spinal cord (from the top of C1 vertebral body to approximately T3, just below the lowest slice level that has PTV in it), brainstem, middle ears, bilateral parotid glands, mandible, and the esophagus (from the bottom of cricoid cartilage to the thoracic inlet). The treatment planning addressed the targets coverage objectives as the highest priority, whereas the organs at risk dosimetric goals were secondary, with the exception of maximal spinal cord and braistem dose. The dose constraints for the organs at risk are summarized in Table 1. Several iterations were performed for optimization, aiming to reduce the mean dose to the larynx to the lowest 
possible, while maintaining the target coverage and the constraints to the other organs at risk according to the dose constraints and priority levels of the target volumes and organs at risk.

Dose-volume histograms

The dose-volume histograms (DVHs) of the target volumes and of the organs at risk were analyzed with the emphasis on the target volumes coverage and the laryngeal dose.

\section{Statistical methods}

A Wilcoxon sign rank test was employed to determine if there was a statistically significant difference between target coverage and laryngeal dose levels achieved for WF-IMRT versus J-IMRT technique. In this study p-values less than 0.05 were considered significant.

\section{RESULTS}

The averaged V100 for the PTV57.6 was $97.7 \%$ for WF-IMRT technique when compared with $88.0 \%(p=0.005)$ for J-IMRT, suggesting a better coverage of PTV57.6 by $100 \%$ isodose line with WF-IMRT. The averaged V95 for the PTV57.6 was $99.2 \%$ for WF-IMRT technique when compared with $97.4 \%(p=0.02)$ for J-IMRT. The averaged V100 for the PTV64 was $98.5 \%$ for WF-IMRT technique when compared with $94.4 \%(p=0.24)$ for J-IMRT. The averaged V95 for the PTV64 was $99.9 \%$ for WF-IMRT technique when compared with $98.9 \%(p=0.02)$ for J-IMRT. The averaged V100 for the PTV70 was $97.4 \%$ for WF-IMRT technique when compared with 94.1\% $(p=0.12)$ for J-IMRT. The averaged V95 for the PT70 was $100.0 \%$ for WF-IMRT technique when compared with $99.5 \%(p=0.04)$ for J-IMRT. Averaged dosimetrical parameters to the target volumes and the organs at risk are represented in Table 2 . The dose volume histograms for the target volumes (PTV57.6) are represented in Figure 1a. 
The averaged mean laryngeal dose was 17.6 Gy for WF-IMRT technique when compared with 18.2 Gy $(p=0.44)$ for J-IMRT. See Table 2. The dose volume histograms for the larynx are represented in Figure 1b.

Moreover, only $40 \%$ of the laryngeal volume received approximately 18 Gy with both IMRT techniques. Only $10 \%$ of the laryngeal volume receives $\geq 20$ Gy with WF-IMRT. See Figure $1 \mathrm{~b}$.

The averaged mean dose within the matchline volumes were 69.3Gy (SD \pm 1.9$)$ for WF-MRT vs 66.2 Gy $(S D \pm 5.13)$ for J-IMRT $(p=0.03)$.

\section{DISCUSSION}

The use of IMRT has been shown to unnecessarily distribute higher dose of radiation to the larynx when the larynx sparing is not attempted as part of the IMRT treatment planning $[5,9,10]$. To avoid this situation, Amdur et al. used a J-IMRT technique with a superior-neck IMRT matched with a conventional anterior lower neck field at the level of the thyroid notch, for patients with non-laryngeal head and neck cancer. Amdur et al. demonstrated that the dose to the normal larynx can be substantially reduced by shielding this organ on an anterior lower neck field [5]. For the WF-IMRT that included both the primary site and the upper and lower cervical lymph nodes, the mean laryngeal dose was 35 Gy in this study. This dose was much higher than the mean laryngeal dose of 17 Gy obtained when the normal larynx was shielded in the conventional anterior lower neck field by using a J-IMRT technique [5]. However, our study revealed that the mean laryngeal dose may be significantly reduced using WF-IMRT, when the larynx is defined as an organ at risk and is incorporated into the IMRT optimization process, without compromising the target volumes coverage or the accomplishment of dosimetric goals elsewhere in the treatment plan. The averaged mean 
laryngeal dose was 18 Gy using WF-IMRT, identical with the averaged mean laryngeal dose achieved with J-IMRT. Furthermore, the current study results suggest that laryngeal doses obtained by using WF-IMRT may be further reduced to values below 25 Gy previously reported in the literature [11]. Webster et al. demonstrated that the dose to the larynx was decreased significantly from a reported mean dose of approximately 45-50 Gy, usually described when larynx sparing is not attempted with WF-IMRT, to a mean dose of $25-40$ Gy if the larynx is considered an organ at risk and is incorporated into the WF-IMRT optimization process [9-11].

Studies evaluating the dose distribution close to the matchline between the opposed lateral fields and the anterior lower neck field as used by conventional radiotherapy for the treatment of non-laryngeal head and neck cancers showed that there may be a few millimeters of increased or decreased dose at or near field junction in the neck [12]. The clinical significance of these dosimetric findings remains unclear. Bubenzer et al. showed, using thermolaminescent dosimetry measurements on a Rando phantom, that the dose homogeneity along the matchline and critical structures are of concern if the upper head and neck fields were treated with IMRT and the lower neck with conventional (supraclavicular) fixed beam using a J-IMRT monoisocentric technique similar to the one employed in our study [13]. The averaged mean dose within the matchline volume were 69.3Gy for WF-MRT vs 66.2 Gy for J-IMRT $(p=0.03)$ in the present study.

Dose inhomogeneity across the matchline has been further investigated and new techniques to mitigate the matchline dosimetrical uncertainties have been explored and reported in the literature [14-16]. Amdur et al. reportedly move the upper border of the anterior lower neck field few millimeters superiorly at least once during the radiotherapy treatment, in order to reduce the dose inhomogeneity at the matchline between upper neck IMRT and conventional anterior lower 
neck field $[14,15]$. Furthermore, a dynamic supraclavicular field-matching technique for head and neck cancer patients treated with IMRT has been developed by the same group [16].

\section{Conclusions}

Taking in consideration the limitation of the present study including a small number of patients, our results suggest that the WF-IMRT technique may achieve an optimal coverage of the target volumes and mean dose to the larynx similar with J-IMRT, while avoiding the dosimetric uncertainty at the matchline present with J-IMRT. Clinical validation is required to determine whether this dosimetric benefit can be translated into meaningful clinical gains for oropharyngeal cancer patients.

\section{REFERENCES:}

1. Mendenhall WM, Parsons JT, Million RR. Unnecessary irradiation of the normal larynx. Int J Radiat Oncol Biol Phys 1990; 18:1531-1533.

2. Lee N, Mechalakos J, Puri DR, et al. Choosing an intensity-modulated radiation therapy technique in the treatment of head-and-neck cancer. Int J Radiat Oncol Biol Phys 2007; 68:1299-1309.

3. Clark $\mathrm{CH}$, Bidmead AM, Mubata CD, et al. Intensity-modulated radiotherapy improves target coverage, spinal cord sparing and allows dose escalation in patients with locally advanced cancer of the larynx. Radiother Oncol 2004; 70:189-198.

4. Kam MK, Chau RM, Suen J, Choi PH, Teo PM. Intensity-modulated radiotherapy in nasopharyngeal carcinoma : dosimetric advantage over conventional plans and feasibility of dose escalation. Int J Radiat Oncol Biol Phys 2003 ; 56(1) :145-157.

5. Amdur RJ, Li JG, Liu C, et al. Unnecessary laryngeal irradiation in the IMRT era. Head Neck 2004; 26:257-263. 
6. Fung K, Yoo J, Leeper HA, et al. Vocal function following radiation for non-laryngeal versus laryngeal tumors of the head and neck. Laryngoscope 2001; 111:1920-1924.

7. Fung K, Yoo J, Leeper HA, et al. Effects of head and neck radiation therapy on vocal function. J Otolaryngol 2001; 30:133-139.

8. Eisbruch A, Schwartz M, Rasch C, et al. Dysphagia and aspiration after chemoradiotherapy for head-and-neck cancer: which anatomic structures are affected and can they be spared by IMRT? Int J Radiat Oncol Biol Phys 2004; 60:1425-1439.

9. Dabaja B, Salehpour MR, Rosen I, et al. Intensity-modulated radiation therapy (IMRT) of cancers of head and neck: Comparison of split-field and whole-field techniques. Int J Radiat Oncol Biol Phys 2005; 63:1000-1005.

10. Fua T, Corry J, Milner A, et al. Intensity modulated radiotherapy for nasopharyngeal carcinomas: Clinical correlation of dose to the pharyngolaryngeal axis and dysphagia. Int J Radiat Oncol Biol Phys 2007; 67:976-981.

11. Webster GJ, Rowbottom CG, Ho KF, et al. Evaluation of larynx-sparing techniques with IMRT when treating the head and neck. Int J Radiat Oncol Biol Phys 2008; 72:617-622.

12. Saw CB, Krishna KV, Enke CA, et al. Dosimetric evaluation of abutted fields using asymmetric collimators for treatment of head and neck. Int $J$ Radiat Oncol Biol Phys 2000; 47:821-824.

13. Bubenzer V, Esfandi M, Jafari N, Manske MA, Wong PF. Dosage along the matchline between upper head-and IMRT fields and conventional supraclavicular fields. Med Dosim 2004; 29(2):104-1108.

14. Amdur R, Liu C, Li J, et al. Matching intensity-modulated radiation therapy to an anterior low neck field. Int J Radiat Oncol Biol Phys 2007; 69:S46-S-48.

15. Li JG, Liu C, Kim S, et al. Matching IMRT fields with static photon field in the treatment of 
head-and-neck cancer. Med Dosim 2005; 30:135-138.

16. Duan J, Shen S, Spencer SA, et al. A dynamic supraclavicular field-matching technique for head-and-neck patients treated with IMRT. Int J Radiat Oncol Biol Phys 2004; 60(3):959-972.

Table 1. Dose constraints for planning organ at risk volumes

\begin{tabular}{|l|l|l|}
\hline PRV & $\begin{array}{l}\text { Mean dose to the } \\
\text { PRV (Gy) }\end{array}$ & $\begin{array}{l}\text { Maximum dose to the } \\
\text { PRV (Gy) }\end{array}$ \\
\hline Spinal cord PRV & & 50 \\
\hline Brain Stem PRV & & 52 \\
\hline Parotid glands PRVs & 26 & 70 \\
\hline Mandible PRV & & \\
\hline Esophagus PRV & $<45$ & \multicolumn{2}{|l|}{} \\
\hline Larynx PRV & Reduce the dose as much as possible. \\
\hline
\end{tabular}

PRV, planning organ at risk volume 
Table 2. Averaged dosimetrical parameters to the target volumes and the organs at risk

\begin{tabular}{|c|c|c|c|c|}
\hline Variable & WF-IMRT & J-IMRT & SD & p value \\
\hline V100 for PTV70 & $97.4 \%$ & $94.1 \%$ & 1.69 & 0.12 \\
\hline V95 for PTV70 & $100 \%$ & $99.5 \%$ & 0.26 & 0.04 \\
\hline V100 for PTV64 & 98.45 & $94.4 \%$ & 2.17 & 0.24 \\
\hline V95 for PTV64 & $99.9 \%$ & $98.9 \%$ & 0.43 & 0.02 \\
\hline $\begin{array}{l}\text { V100 for } \\
\text { PTV57.6 }\end{array}$ & $97.7 \%$ & $88.0 \%$ & 2.21 & 0.005 \\
\hline V95 for PTV57.6 & $99.2 \%$ & $97.4 \%$ & 0.59 & 0.02 \\
\hline $\begin{array}{l}\text { Spinal cord } \\
\text { maximum dose }\end{array}$ & 43.7 Gy & $46.8 \mathrm{~Gy}$ & 1.07 & 0.01 \\
\hline $\begin{array}{l}\text { Esophageal } \\
\text { maximum dose }\end{array}$ & 37.7 Gy & $59.5 \mathrm{~Gy}$ & 1.73 & 0.005 \\
\hline
\end{tabular}




\begin{tabular}{|l|l|l|l|l|}
\hline $\begin{array}{l}\text { Lungs maximum } \\
\text { dose }\end{array}$ & $42.2 \mathrm{~Gy}$ & $57.1 \mathrm{~Gy}$ & 2.53 & 0.01 \\
\hline $\begin{array}{l}\text { Laryngeal } \\
\text { maximum dose }\end{array}$ & $30.4 \mathrm{~Gy}$ & $49.2 \mathrm{~Gy}$ & 3.59 & 0.006 \\
\hline $\begin{array}{l}\text { Laryngeal } \\
\text { minimum dose }\end{array}$ & $14.9 \mathrm{~Gy}$ & $10.3 \mathrm{~Gy}$ & 0.45 & \\
\hline $\begin{array}{l}\text { Laryngeal mean } \\
\text { dose }\end{array}$ & $17.6 \mathrm{~Gy}$ & $18.2 \mathrm{~Gy}$ & 0.84 & \\
\hline
\end{tabular}

Figure 1a. Dose-volume histogram for the target volumes (PTV57.6) using whole-neck field IMRT (WF-IMRT) and junctioned IMRT (J-IMRT) 


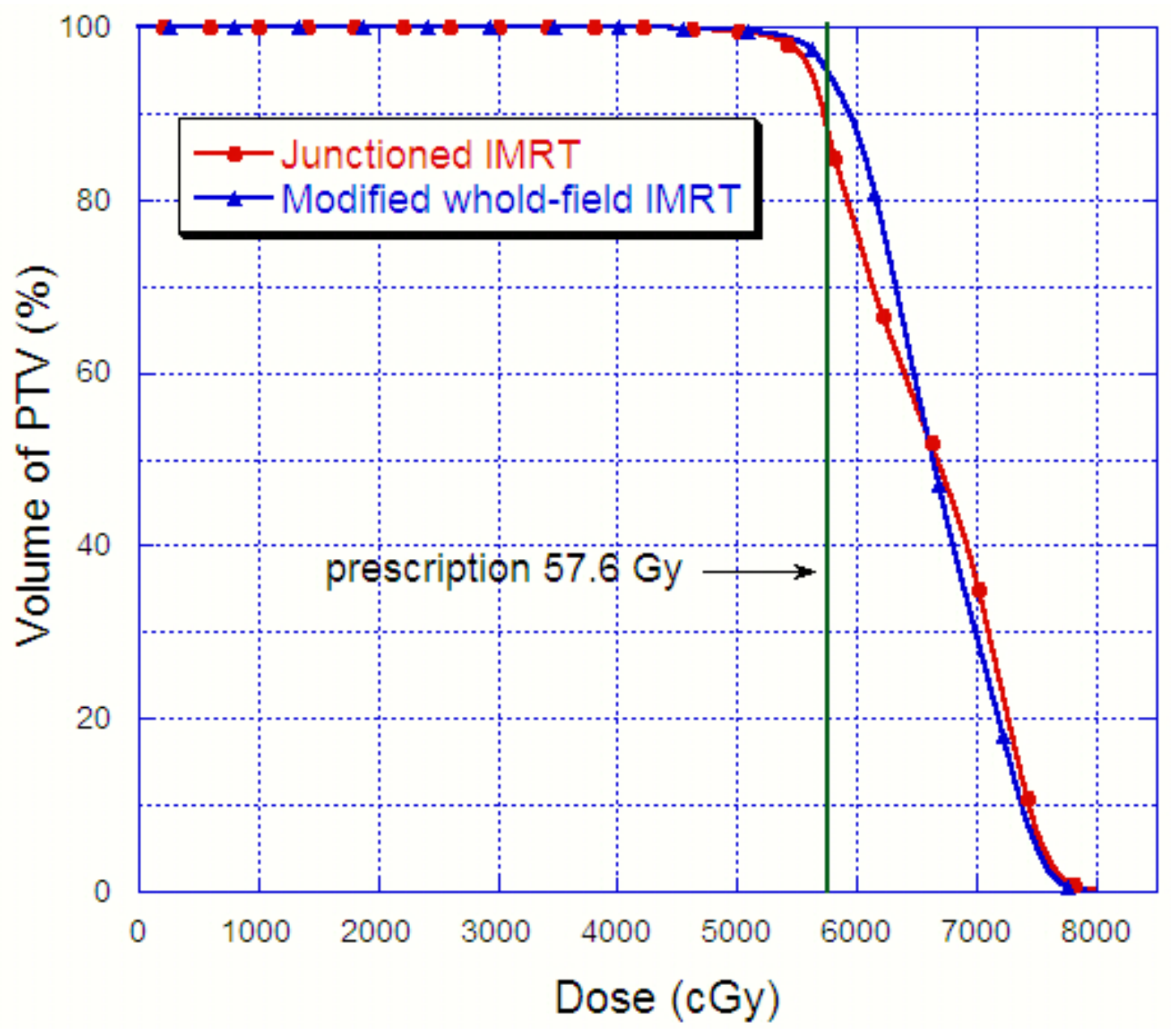

Figure 1b. Dose-volume histogram for the larynx using whole-neck field IMRT (WF-IMRT) and junctioned IMRT (J-IMRT) 


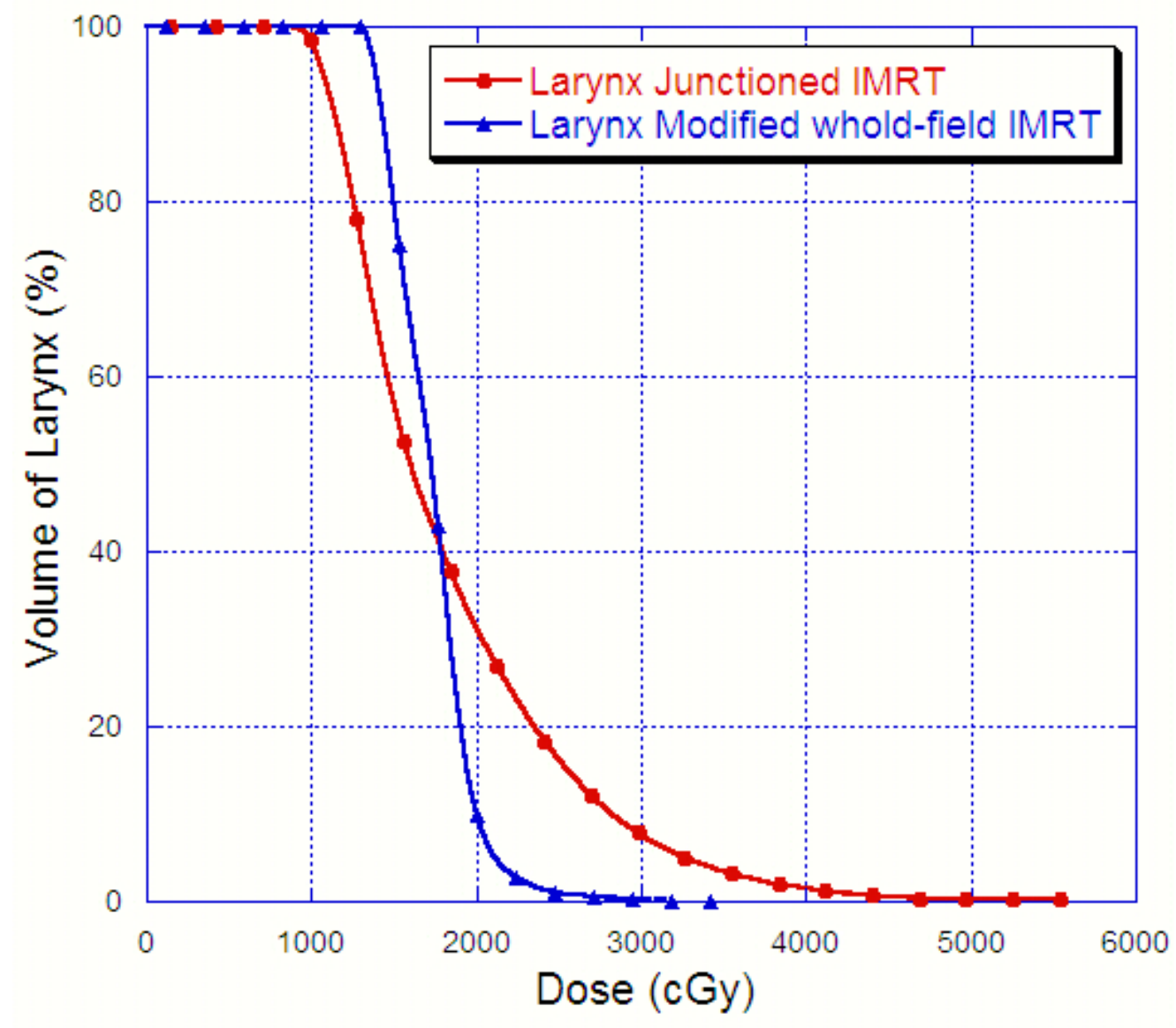

